\title{
Value of Vestibular Evoked Myogenic Potentials in Identifying Different Forms of Endolymphatic Hydrops: A Cross-Sectional Study
}

\author{
HESHAM M. TAHA, M.D.*; IHAB NADA, M.D.**; TAYSEER T. ABDEL RAHMAN, M.D.* and \\ MAHA M. ALAGAMY, M. S c. *** \\ The Department ofENT, Audiology Unit, Faculty of Medicine, Ain Shams University*, Department of ENT, Faculty of Medicine, \\ MUST University** and Department of ENT, Audiology Unit, Faculty of Medicine, MUST University***
}

\begin{abstract}
Background: Meniere's disease is idiopathic Endolymphatic Hydrops. Vestibular evoked myogenic potential is a neurophysiological assessment technique that is used determine the function of the otolith organs of the inner ear. Using vestibular evoked myogenic potential tests may identify the different forms of the Endolymphatic Hydrops.

Aim of Study: The aim of this study is to explore the role of vestibular evoked myogenic potentials (VEMP) in diagnosis of the different kinds of Meniere's disease (MD).

Patients and Methods: Three groups of patients that were selected according to the diagnosis, definitive MD (DMD), cochlear MD (CMD) and recurrent vestibulopathy (RV) group. Basic audiological evaluation was carried out. It included Pure tone audiometry, speech audiometry, acoustic immittance testing and electrocochleography. Otolith function assessment was carried out in the form of:
\end{abstract}

Cervical VEMP (c-VEMP) and ocular VEMP (o-VEMP). Vestibular assessment was carried out: The test protocol included spontaneous nystagmus, gaze test, oculomotor tests, positioning test (Dixhallpike), positional nystagmus, and caloric testing. The three groups were compared for all demographic variables as well as clinic-pathologic variables, and tests results.

Results: The overall sample was 40 cases; 13 with DMD, 10 with CMD and 17 with RV. The mean age is variable between groups ( $p$-value $<0.001$ ) with the highest in the DMD group (38.3 \pm 7.4 years) and the lowest in the CMD group ( $25.2 \pm 4.6$ years). The male to female ratio is variable between groups of the study ( $p$-value=0.03) with the highest ratio 9:4 in the DMD group. There was a significant difference between groups $(p<0.001)$ regarding dizziness. Asymmetric response of c-VEMP was seen in nine (9/13) $(69.2 \%)$ of DMD cases, ( $14 / 17)(82.4 \%)$ of RV cases and only in three (3/10) (30\%) of CMD cases, ( $p$-value $<0.001)$. Low amplitude of o-VEMP was seen in (11/13) $(84.6 \%)$ of DMD cases, $(9 / 10)(90 \%)$ of CMD cases, (15/17) $(88.2 \%)$ of RV cases, and only in two ( $25 \%$ ) of normal cases, ( $p$-value $=0.002)$. A significant difference was found between definitive and cochlear Meniere's as

Correspondence to: Dr. Hesham M. Taha, The Department of ENT, Audiology Unit, Faculty of Medicine, Ain Shams University regards o-VEMP inter-aural amplitude difference (IAD) ( $p$ value $=0.032$ ) only. There was a significant positive strong correlation between age and EchoG sp/ap ratio left ear, oVEMP IAD and o-VEMP left ear $\mathrm{n} 1$. In addition, there is a significant positive weak correlation between duration and EchoG sp/ap ratio left ear and c-VEMP left ear $n 1$.

Conclusion: Asymmetric response of c-VEMP can be used as a diagnostic tool to differentiate different kinds of MD: DMD, RV and CMD

Key Words: Meniere's disease - Ocular vestibular evoked myogenic potentials - Cervical vestibular evoked myogenic potentials - Definitive Ménière's Cochlear Ménière's - Recurrent vestibulopathy.

\section{Introduction}

MENIERE'S disease (MD), one of the most common disorders of the inner ear, is thought to be an idiopathic progressive disease. It is caused by hair cells degeneration in the cochlea and vestibule. Prosper Meniere was the scientist who described it; thus, it was named after him. While on the other hand Ménière' syndrome can be secondary to various processes interfering with the normal production or resorption of the endolymph like trauma, viral vestibular ganglionitis, and genetic predisposition. Also, it is thought to be caused by pathologies of the stria vascularis that can result in its symptoms [1,2].

The excess hydraulic pressure within the inner ear's endolymphatic system causes unstable hearing loss, occasional vertigo, tinnitus or ringing in the ears, and aural fullness. The term endolymphatic hydrops can be synonymously used with MD and Ménière's syndrome [3].

Ocular vestibular evoked myogenic potentials (o-VEMP) and cervical vestibular evoked myogenic 
potentials (c-VEMPs) are both elicited by acoustic stimulation of the vestibular organs. The response is measured in muscle EMG. The o-VEMP is recorded by placing recording electrodes below the eyes and maintaining an upward gaze during recording, forcing the inferior oblique muscle closer to the recording electrode. One major difference between the o-VEMP and c-VEMP, is that the oVEMP is a contralateral response whereas the cVEMP is ipsilateral. In addition, the c-VEMP is an inhibitory response and the o-VEMP is an excitatory response. It has been also thought that the o-VEMP is mainly mediated by utricular stimulation while the c-VEMP is a saccular response [4].

There is little written on c-VEMP and o-VEMP in different category of MD: Definitive Ménière's ( DMD), cochlear Ménière's (CMD) and recurrent vestibulopathy (RV). Thus, the rationale intended for this current study was to explore the role of VEMP in diagnosis of the different kinds of MD.

\section{Patients and Methods}

The current study was a cross-sectional study that included all patients aged 18 years or more with MD of any degree who came to the outpatient clinic of Al Demerdash Hospital, Ain Shams University, Cairo, Egypt, during the period from March 2018 and March 2020. The objective of the study as well as the steps of the procedures were explained plainly to the study participants. All participants included in the study have provided an informed consent. The Ethical Review Board has approved this study.

The patients were divided into three groups and were selected according to the diagnosis. Group 1 were patients with DMD. Idiopathic endolymphatic hydrops in patients having the classic tetrad of MD which are at least two episodic vertiginous spells lasting 20 minutes or longer, tinnitus, ear fullness, and fluctuating hearing. Group 2 were patients with RV. Those patients were characterized by more than a single episode of vertigo of duration characteristic of that occurring with hydrops but without auditory or neurological symptoms or signs. Group 3 were patients with CMD. Those with fluctuating sensorineural hearing loss with tinnitus. Exclusion criteria were: Refusal to participate, patients with sudden SNHL, patients with retrocochlear pathology, patients with neurological disorders, patients with any history suggestive of vestibular neuritis, any patients with history suggestive of head trauma, or patients with any history suggestive of henion nositional naroxvsmal attacks.
Full history was taken from all patients included duration of the condition, onset, course, fluctuation, side of hearing loss, character of tinnitus, frequency, duration \& severity of vertiginous attacks, presence of aural fullness. History of medical treatment and its effect on the control of the condition, and history of ear surgery was also emphasized. History of precipitating factors for endolymphatic hydrops was emphasized too.

Also there was a control group that consisted of 8 patients age between 25-40 years of age. Regarding gender; $4 / 8$ females, and 4/8 females. They had normal middle ear function and within normal audiograms and speech discrimination scores.

Basic audiological evaluations were carried out. It included the following procedures:

Pure tone audiometry: Air conduction thresholds were tested at the following frequencies 0.25 , $0.5,1,2,4$ and $8 \mathrm{KHz}$. Bone conduction thresholds were tested at $0.5,1,2$ and $4 \mathrm{KHz}$. Effective masking using narrow band noise was introduced to the contralateral ear whenever indicated. Two channel pure tone audiometry (AC 40) was used.

Speech audiometry: This included speech perception threshold testing (SRT), and word discrimination testing at $40 \mathrm{~dB}$ sensation level (ref SRT) or at most comfortable levels in a sound treated room.

Acoustic immittance testing: It included tympanometry and acoustic reflex threshold measurement.

Electrocochleography: Non-invasive tympanic membrane electrocochleography was carried out using Otopront Evoked Potential System. The active electrode was Gold plated Triptode by Biologic Corporation. Sound delivery system was insert phone placed in the external auditory canal. The other two electrodes were placed at the contralateral mastoid and the forehead to serve as negative and ground electrodes, respectively. Stimuli consisted of 100 microsecond clicks were generated at a rate of 7.1 pulses/second at $90 \mathrm{db} \mathrm{nHL}$. Alternating polarity was used to cancel the cochlear microphonic and the stimulus artifacts. A system bandpass of $10 \mathrm{HZ}$ to $1.5 \mathrm{kHZ}$ was utilized. Analysis of the waveforms was carried out by measuring the amplitude of the SP from the pre-stimulus baseline to the shoulder of the preceding edge of the AP. The AP amplitude was measured from the pre-stimulus baseline to the maximum peak of $\mathrm{N} 1$ ( Fig. 1). 


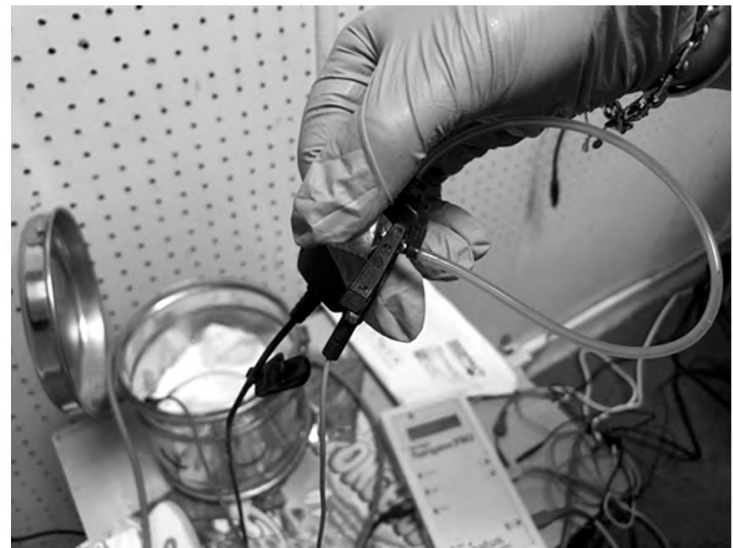

Fig. (1A): Stimuli consisted of 100 microsecond clicks, generated at a rate of 7.1 pulses/second at $90 \mathrm{db}$ nHL. Alternating polarity was used to cancel the cochlear microphonic and the stimulus artifacts. A system bandpass of $10 \mathrm{HZ}$ to $1.5 \mathrm{kHZ}$ was utilized.

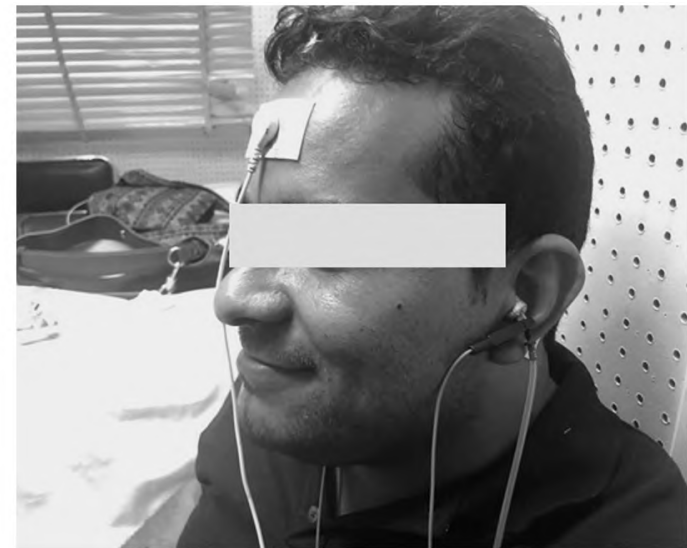

Fig. (1B): The active electrode was Gold plated Triptode by Biologic corporation. Sound delivery system was insert phone placed in the external audifigure. The other two electrodes were placed at the contralateral mastoid and the forehead to serve as negative and ground electrodes respectively.

Fig. (1): Noninvasive tympanic membrane Electrocochleography settings.

\section{Otolith function assessment was carried out in the form of: \\ Cervical vestibular evoked myogenic potentials $(c-V E M P)$ :}

The subject was in a sitting position and was instructed to rotate his head to the opposite side of the stimulated ear and to maintain muscle contraction. Otopront evoked potential system was used. The silver surface electrodes were placed over the midpoint of the sternomastoid muscle ( active one), a reference electrode was placed over the upper sternum, and the ground electrode was placed at the forehead. The electrode impedance was kept under five uhms. C-VEMP testing was recorded bilaterally with $500 \mathrm{HZ}$ tone burst stimulus, rise/fall time of two millisecond and a plateau of one millisecond. The repetition rate was five per second at $90 \mathrm{~dB}$ nHL intensity using an insert receiver. After that, the EMG response from each side was amplified with band pass filter (10$1500 \mathrm{HZ}$ ). Two hundred sweeps were averaged with a window of 50 milliseconds. At least two consecutive averages were recorded from each side to verify reproducibility. The average of two runs was taken for the amplitude and latencies, and compared to the values of the control group. Latencies of p13, N23 were measured. P13 is the first positive peak of c-VEMP and n23 is the first negative peak following p13. For the evaluation of the amplitude, the inter peak p13-n23 amplitude were measured. The adult normative data of audiology unit Ain shams was $15.5( \pm 2.3)$ and 23.6
$( \pm 2.9)$ milliseconds for the latency of the p13 and n23 waves, respectively. For air conduction stimulus, p13-n23 inter amplitude was $112( \pm 67.5)$ milliseconds $[\mathbf{5 , 6 ]}$.

Ocular vestibular evoked myogenic potentials $(o-V E M P)$ :

The subject was in the sitting position and was instructed to look upward to a small fixed target more than two meters. The vertical eye position was at angle of approximately 30-35 degree above the horizontal line. The surface electrodes were placed at three mm below the eye and centered beneath their pupil not the inferior ocular muscle, a reference electrode was placed on the chin and the ground impedance was kept under five uhms as $\mathrm{n} 1$ responses are best recorded from the extra-ocular muscles slightly below the eye contralateral to stimulation $[\mathbf{5 , 6 ]}$.

O-VEMP testing was recorded bilaterally with the $500 \mathrm{HZ}$ tone burst stimulus, rise/fall time of two millisecond and a plateau of one millisecond. Repletion rate was $5 / \mathrm{sec}$ at $90 \mathrm{~dB}$ nHL intensity using an insert receiver. The EMG response from each side was amplified by band pass filter (101500HZ). Two-hundred sweeps was averaged with a window of 50 millisecond. At least two consecutive averages were recorded from each side to verify reproducibility. The average of two runs was taken for the amplitude and latencies were compared to the values well known. Latencies of $n 1$, p1 were measured. N1 is the first nerative naak 
of o-VEMP and p1 is the first positive peak following n1. For evaluation of the amplitude, n1-p1 inter amplitude was measured. The latency of the $\mathrm{n} 1$ and $\mathrm{p} 1$ from the adult normative data of audiology unit Ain Shams University was $11.1( \pm 0.4)$ and $15.9( \pm 0.4)$ milliseconds respectively for air conduction stimuli. N1-P1 inter-amplitude was $14.6( \pm 4.80)$.

\section{Vestibular assessment was carried out:}

Video Nystagmography (VNG): The test protocol included spontaneous nystagmus, gaze test, oculomotor tests, positioning test (Dix-hallpike), positional nystagmus, and caloric testing. Video Nystagmography (VNG) was carried out using Micromedical Tech, Meta 4 software version 4.5.

For the caloric testing, otological examination was done first to make sure that there was not a condition that would hinder water irrigation feasibility. Then, patient was positioned on his back, with the head 30 degrees above horizontal line and that pre-caloric nystagmus was observed if present and was measured. The patient was informed about the steps of the test and the possibility of vertigo to occur. Fixation was then introduced to calculate the fixation index.

For the alternate binaural bi-thermal caloric testing, we started irrigation of the ear with $200 \mathrm{cc}$ water at 44 degrees over 40 seconds. Then, the patient was instructed to keep his eyes opened and chin up. After that, we gave the patient mental tasks, after recording for 40-50 seconds, the patient was tested for fixation index. The goggles were then opened, and testing was stopped until nystagmus has completely stopped, and the patient no more had vertigo. The same steps were repeated to the other ear, and those were the steps for the warm test. As for the cool test both ears were irrigated with 30 degrees water. The software recorded and measured the total response and fixation index of nystagmus, following each irrigation. Also, the software inserted right and left total response in Jongkees' formula and output the response of one ear relative to the other in percentile terms. Jongkees' formula to calculate the asymmetry between the ears [8]:

Percentage of asymmetry $=(R C+R W)-(L C+L W) X 100$

$$
(\mathrm{RC}+\mathrm{RW}+\mathrm{LC}+\mathrm{LW})
$$

Where RC, RW, LC, and LW are peak slow phase velocity for the responses to right cool, right warm, left cool, and left warm irrigations, respectively.

\section{Statistical analysis:}

The patients were grouped into three groups, and a control group. The groups were compared for all demographic variables as well as clinicpathologic variables and for all tests. Recorded data were coded, entered, cleaned, and analyzed using the statistical package for social sciences, version 25.0 (SPSS Inc., Chicago, Illinois, USA). Quantitative data were expressed as mean \pm standard deviation (SD) or median and interquartile range ( IQR). Qualitative data were expressed as frequency and percentage. Chi-square $\left(\mathrm{x}^{2}\right)$ test of significance or ANOVA was used in order to compare proportions between the qualitative parameters, and the student $t$-test was used for the numerical parametric data. Non-parametric statistical tests were applied whenever indicated. Post-hoc tests were made for further comparisons. The confidence level was set to $95 \%$ and the margin of error accepted was set to $5 \%$. So, the $p$-value was considered significant if $<0.05$. Correlation Coefficient was calculated.

\section{Results}

The study included 40 patients with different degrees of MD: 13 with DMD, 10 with CMD and 17 with RV. In addition, the study included eight normal control subjects. Therefore, the total sample was 48 subjects.

Demographic and clinic -pathologic characteristics of groups:

The mean age was $32.9 \pm 6.8$ years with a minimum age of 20 and a maximum of 47 years. The mean age is variable between groups ( $p$-value $<0.001$ ) with the highest in the DMD group (38.3 \pm 7.4 years) and the lowest in the CMD group (25.2 \pm 4.6 years), as shown in Table (1).

Nineteen $(39.6 \%)$ of cases was males and 29 ( $60.4 \%)$ was females. The male to female ratio is variable between groups of the study ( $p$-value $=0$. 03) with the highest ratio 9:4 in the DMD group. Post-hoc testing revealed that male gender is more prevalent in the DMD group than other groups $(p=0$. $002)$. Female gender is more prevalent in the RV group ( $p=0.009$ ), as shown in Table (1). Regarding positive family history, there is a significant difference between groups $(p<0.001)$. Posthoc testing revealed that positive family history is not related to CMD $(p<0.001)$. Vertigo is more prevalent in the RV than other groups $(p=0.002)$. Hearing loss is more prevalent in the CMD group ( $p<0.001)$, as shown in Table (1).

There is a significant difference between groups ( $p$ $<0.001)$ regarding dizziness. Post-hoc testing 
revealed that the CMD group $(p<0.001)$ has the least frequency in sensation of imbalance or rotation than the two other groups. Also, the prevalence of headache is significantly different between groups ( $p$ $<0.001)$. Post-hoc testing revealed that headache is less prevalent in the CMD group $(p<0.001)$ than other groups. In addition, aural fullness is less prevalent in the CMD group $(p<0.001)$ than other groups. Tinnitus is less prevalent in the CMD group ( $p$ $<0.001)$ than other groups. Regarding history of Tumarkin crisis, there is no significant difference between groups ( $p=0.09$ ), as shown in Table (1).

Five cases $(38.5 \%)$ of DMD and six cases $(60 \%)$ of CMD have had unilateral affection; while, 16 cases $(94.1 \%)$ of RV have had unilateral affection. There is a significant difference between groups with regard laterality of the condition $(p<0.001)$. Post-hoc tests showed that Unilateral affection is more in RV group and CMD while bilateral affection is more in the DMD group $(p<0.001)$. The duration is different in the three study groups $(p$ $=0.011$ ). Post-hoc tests showed that DMD group has significantly longer duration than other groups ( $p$ $<0.001$ ), as shown in Table (1).

\section{Basic audiological evaluation:}

After acoustic emittance testing, all cases in all groups have had normal tympanograms (right and left). The type of hearing loss is variable between groups $(p<0.001)$. All cases of DMD 13 ( $100 \%)$ and CMD $10(100 \%)$ have had SNHL, with only two cases (11.8\%) of RV. Post-hoc testing revealed that SNHL is significantly higher in $\mathrm{DMD}(p$ $=0.001)$ and CMD $(p=0.005)$ than in RV group, as shown in Table (1). The right ear audiogram is significantly $(p<0.001)$ affected in the DMD and the CMD groups than other groups especially at $250,500 \& 1000 \mathrm{HZ}$ frequencies. Also, the left ear audiogram showed highly significant affection in the DMD and the CMD groups than other groups especially at $250,500,1000 \& 2000 \mathrm{HZ}$ frequencies, as shown in Figs. $(2,3)$. There is highly significant difference between the three groups regarding the left ear and right ear speech discrimination score. Post-hoc tests showed that the scores are significantly $(p<0.001)$ lower in the DMD group than both in CMD group and the RV group, as shown in Table (2).

Table (1): Demographic and clinical characteristics of cases.

\begin{tabular}{|c|c|c|c|c|}
\hline & $\begin{array}{l}\text { DMD } \\
\mathrm{N}=13\end{array}$ & $\begin{array}{l}\text { CMD } \\
\mathrm{N}=10\end{array}$ & $\begin{array}{c}\mathrm{RV} \\
\mathrm{N}=17\end{array}$ & $p$-value \\
\hline Male: Female ratio & 9:04 & 3:07 & $3: 14$ & 0.03 \\
\hline Age, years mean \pm SD & $38.3 \pm 7.4$ & $25.2 \pm 4.6$ & $33.8 \pm 3.6$ & $<0.001$ \\
\hline Positive family history, n (\%) & $12(92.3)$ & $1(10.0)$ & $14(82.4)$ & $<0.001$ \\
\hline \multicolumn{5}{|l|}{ Main complaint, $n(\%)$ : } \\
\hline Hearing loss & $1(7.7)$ & $9(90.0)$ & $0(0.0)$ & \multirow[t]{2}{*}{$<0.001$} \\
\hline Vertigo & $12(92.3)$ & $1(10.0)$ & $17(100.0)$ & \\
\hline \multicolumn{5}{|l|}{ Dizziness, $n(\%)$ : } \\
\hline No dizziness & $0(0.0)$ & $9(90.0)$ & $0(0.0)$ & \multirow[t]{4}{*}{$<0.001$} \\
\hline Sense of rotation of surrounding & $3(23.1)$ & $1(10.0)$ & $8(47.1)$ & \\
\hline Sense of imbalance & $8(61.5)$ & $0(0.0)$ & $9(52.9)$ & \\
\hline Sense of self rotation & $2(15.4)$ & $0(0.0)$ & $0(0.0)$ & \\
\hline Headache, n (\%) & $13(100.0)$ & $3(30.0)$ & $16(94.1)$ & $<0.001$ \\
\hline Aural fullness, n (\%) & $13(100.0)$ & $1(10.0)$ & $16(94.1)$ & $<0.001$ \\
\hline Tinnitus, n (\%) & $13(100.0)$ & $4(40.0)$ & $15(88.2)$ & 0.001 \\
\hline History of tumarkin crisis, $\mathrm{n}(\%)$ & $5(38.5)$ & $0(0.0)$ & $4(23.5)$ & 0.09 \\
\hline \multicolumn{5}{|l|}{ Laterality, $n(\%)$ : } \\
\hline Unilateral & $5(38.5)$ & $6(60)$ & $16(94.1)$ & \multirow[t]{2}{*}{$<0.001$} \\
\hline Bilateral & $8(61.5)$ & $4(40)$ & $1(5.9)$ & \\
\hline \multicolumn{5}{|l|}{ Duration of the disease: } \\
\hline Mean $\pm \mathrm{SD}$ & $27.5 \pm 22.8$ & $10.0 \pm 3.8$ & $13.8 \pm 6.0$ & \multirow[t]{2}{*}{0.011} \\
\hline Median (IQR) & $24.0(24.0)$ & $12.0(6)$ & $12.0(8.0)$ & \\
\hline \multicolumn{5}{|l|}{ Type of hearing loss, $n(\%)$ : } \\
\hline SNHL & $13(100)$ & $10(100)$ & $2(11.8)$ & \multirow[t]{2}{*}{$<0.001$} \\
\hline No hearing loss & $0(0.0)$ & $0(0.0)$ & $15(88.2)$ & \\
\hline
\end{tabular}




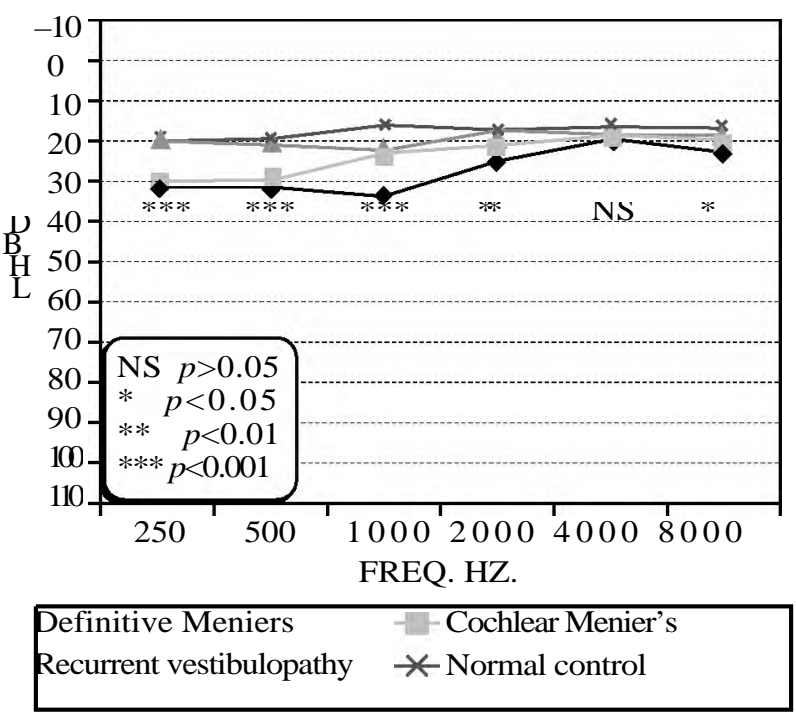

Fig. (2): Right Ear Audiogram.

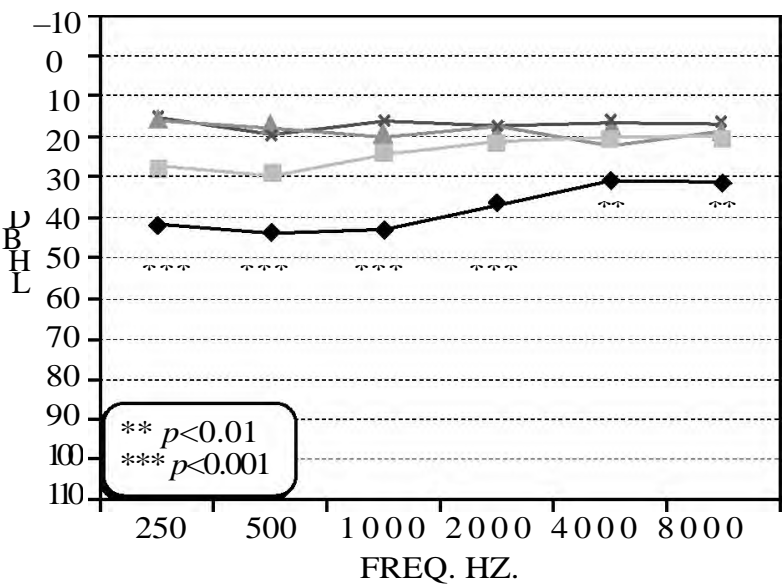

Definitive Meniers Cochlear Menier's Recurrent vestibulopathy $\quad *$ Normal control

Fig. (3): Left Ear Audiogram.

Table (2): Speech discrimination score and electrocochleography and vestibular assessment.

\begin{tabular}{|c|c|c|c|c|c|}
\hline & $\begin{array}{l}\text { DMD } \\
\mathrm{N}=13\end{array}$ & $\begin{array}{l}\text { CMD } \\
\mathrm{N}=10\end{array}$ & $\begin{array}{c}\mathrm{RV} \\
\mathrm{N}=17\end{array}$ & $\begin{array}{c}\text { Normal control } \\
\mathrm{N}=8\end{array}$ & $\begin{array}{c}p- \\
\text { value }\end{array}$ \\
\hline \multicolumn{6}{|l|}{ Speech discrimination score: } \\
\hline Right ear, mean \pm SD & $93.2 \pm 6.6$ & $100.0 \pm 0.0$ & $99.8 \pm 1.0$ & $100.0 \pm 0.0$ & $<0.001$ \\
\hline Left ear, mean \pm SD & $87.1 \pm 3.9$ & $96.4 \pm 2.3$ & $98.4 \pm 2.0$ & $99.0 \pm 4.3$ & $<0.001$ \\
\hline \multicolumn{6}{|l|}{ Right ear ECochG sp/ap ratio: } \\
\hline Mean \pm SD & $0.43 \pm 0.18$ & $0.45 \pm 0.08$ & $0.48 \pm 0.19$ & $0.36 \pm 0.03$ & 0.346 \\
\hline Abnormal, n (\%) & $5(38.5)$ & $5(50.0)$ & $6(35.6)$ & $0(0.0)$ & 0.746 \\
\hline \multicolumn{6}{|l|}{ Left ear ECochG sp/ap ratio: } \\
\hline Mean \pm SD & $0.70 \pm 0.07$ & $0.48 \pm 0.15$ & $0.54 \pm 0.13$ & $0.34 \pm 0.04$ & $<0.001$ \\
\hline Abnormal, n (\%) & $11(84.6)$ & $7(70.0)$ & $10(58.8)$ & $0(0.0)$ & 0.311 \\
\hline Positiona nystagmus, n (\%) & $6(46.2)$ & $3(30)$ & $9(52.9)$ & $0(0.0)$ & 0.07 \\
\hline Asymmetric caloric test, $\mathrm{n}(\%)$ & $2(15.4)$ & $0(0.0)$ & $2(11.8)$ & $0(0.0)$ & 0.43 \\
\hline
\end{tabular}

\section{Electrocochleography:}

Non-invasive tympanic membrane electrocochleography was performed for all cases. The mean sp/ap ratio was $>0.40$ in all study groups except for normal cases, as shown in Table (2). There is highly significant ( $p$-value $<0.001$ ) difference in the mean left ear sp/ap ratio between groups. Posthoc tests for multiple comparisons revealed a significant difference between DMD and CMD ( $p$ value $<0.001)$ and between the DMD group and the RV group ( $p$-value $=0.003$ ). However, no differences could be detected in the right ear sp/ap ratio between groups, as shown in Table (2). $V N G)$ :

Vestibular assessment: Video-Nystagmography (

Classic Video-Nystagmography VNG test battery was performed searching for spontaneous, gaze evoked positional and positioning nystagmus. Oculomotor test battery included regular random saccade testing, together with eye tracking and optokinetic tests. Bithermal Caloric test was performed. All cases have had a normal VNG spontaneous nystagmus test, VNG gaze test, VNG saccade test, tracking test, optokinetic test and Dix hallpike maneuver. Positional nystagmus and caloric test were comparable between all study groups ( $p$ value $>0.05$ ), as shown in Table (2).

\section{Otolith function assessment:}

Asymmetric response of c-VEMP was seen in nine $(69.2 \%)$ of DMD cases, $14(82.4 \%)$ of RV cases and only in three (30\%) of CMD cases, ( $p$ value $<0.001$ ), as shown in Table (3). There is highly significant $(p<0.001)$ difference in the mean of c-VEMP between groups. Post-hoc test for multiple comparisons revealed a significant difference between DMD group and normal control ( $p$ value $<0.001)$ and between the RV group and the normal control $(p=0.002)$. There is a significant 
difference ( $p$-values $<0.001)$ in $\mathrm{p} 1 \& \mathrm{n} 1$ latencies in right ear between the study groups, as shown in Table (3). Post-hoc testing showed that in the right ear $\mathrm{p} 1$ latency, the difference is between $\mathrm{RV}$ and both DMD $(p<0.001)$ and CMD $(p<0.001)$. In addition, the right ear $\mathrm{n} 1$ latency, the difference is between RV and both DMD $(p<0.001)$ and CMD $(p$ $=0.024)$. $\mathrm{c}$-VEMP IAD was significantly variable between groups $(p=0.028)$, it was $0.13 \pm 0.02$ in the DMD, $0.13 \pm 0.01$ in the CMD \& $0.24 \pm 0.17$ in the RV groups. Post-hoc testing revealed nonsignificant results.

Low amplitude of o-VEMP was seen in 11 ( 84.6\%) of DMD cases, 9 (90\%) of CMD cases, $15(88.2 \%)$ of RV cases, and only in two $(25 \%)$ of normal cases, $(p$-value $=0.002)$. Latencies of $n 1$ and $\mathrm{p} 1$ were measured in right and left ear in all cases. There is a significant difference in right ear p1 ( $p$-values $=0.007) \&$ left ear p1 ( $p$-values $=0.027)$ latencies between the study groups, as shown in Table (3). o-VEMP IAD was significantly variable between groups $(p=0.018)$, it was $0.39 \pm 0.08$ in the DMD, $0.17 \pm 0.06$ in the CMD \& $0.35 \pm 0.07$ in the
RV groups. Post-hoc testing showed no differences between groups. Post-hoc tests for multiple comparisons revealed a significant difference between definitive and cochlear Meniere's as regard oVEMP inter-aural amplitude difference (IAD) ( $p$ value $=0.032$ ) only, as shown in Table (4).

\section{Further analyses:}

Further analyses were carried out to explore the relationship between the duration of symptoms and the laterality of the condition. The duration of symptoms was not significantly $(p=0.068)$ different according to laterality of the condition. The median ( IQR) duration was $12.0(2.0)$ years in the unilateral versus 12.0 (24) in the bilaterally affected cases. Also, correlations between age, duration and the different tests findings were carried out. There is a significant positive strong correlation between age and EchoG sp/ap ratio left ear, oVEMP IAD and o-VEMP left ear $\mathrm{n} 1$. In addition, there is a significant positive weak correlation between duration and EchoG sp/ap ratio left ear and c-VEMPleft ear $\mathrm{n} 1$, as shown in Table (5).

Table (3): Cervical and ocular vestibular evoked myogenic potentials.

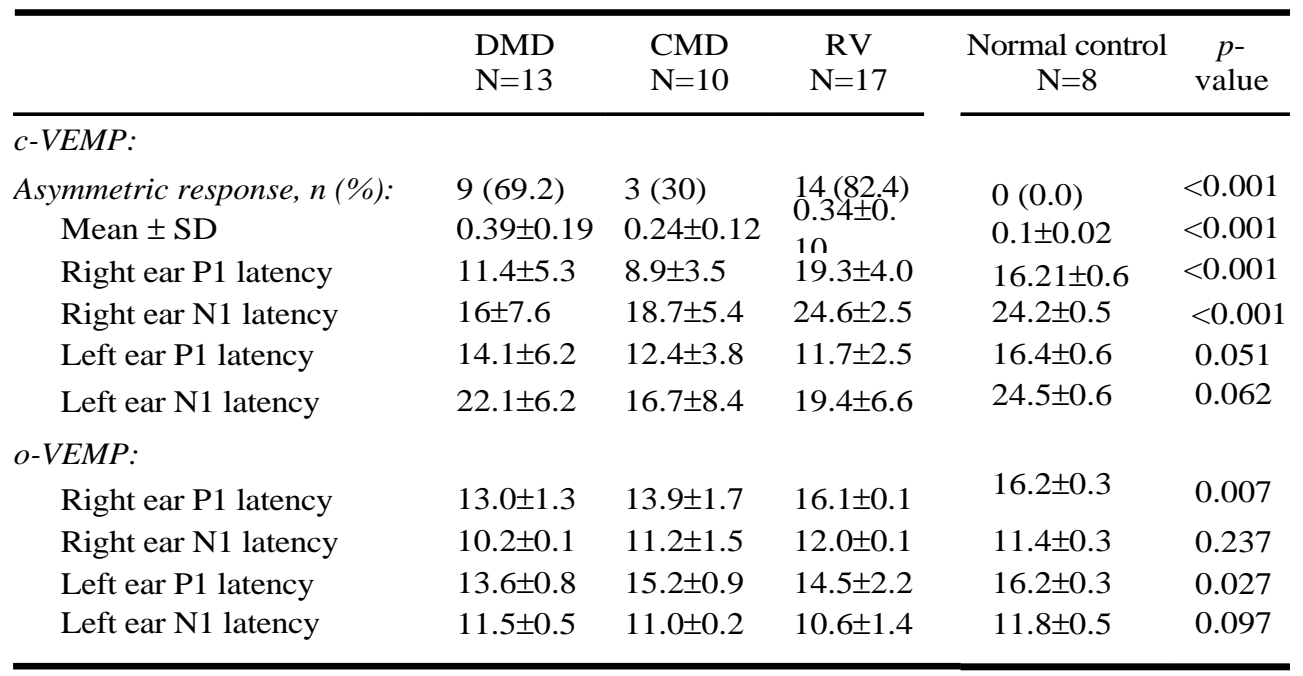

Table (4): IAD of c-VEMP and o-VEMP.

\begin{tabular}{lcllc}
\hline & DMD & CMD & RV & $p$-value \\
\hline Abnormal c-VEMP IAD, n (\%) & $7(53.8)$ & $0(0.0)$ & $3(17.6)$ & 0.008 \\
Abnormal o-VEMP IAD, n (\%) & $2(100.0)$ & $0(0.0)$ & $1(50.0)$ & 0.028 \\
c-VEMP IAD, mean \pm SD & $0.13 \pm 0.020 .13 \pm 0.01$ & $0.24 \pm 0.17$ & 0.101 \\
o-VEMP IAD, mean \pm SD & $0.39 \pm 0.080 .17 \pm 0.06$ & $0.35 \pm 0.07$ & 0.018 \\
\hline
\end{tabular}

Post-hoc tests revealed difference between DMD \& CMD in the o-VEMP only. 
Table (5): Correlations between age, duration and all tests findings.

\begin{tabular}{llllc}
\hline & \multicolumn{2}{c}{ Age } & \multicolumn{2}{c}{ Duration } \\
\cline { 2 - 5 } & $\begin{array}{l}\text { Correlation } \\
\text { coefficient }\end{array}$ & $p$-value & $\begin{array}{l}\text { Correlation } \\
\text { coefficient }\end{array}$ & $p$-value \\
\hline ECochG sp/ap ratio right ear & -0.17 & 0.305 & 0.27 & 0.096 \\
ECochG sp/ap ratio left ear & $0.51^{*} *$ & 0.001 & $0.34^{*}$ & 0.03 \\
OVEMP IAD & $0.91 * *$ & 0.002 & 0.66 & 0.073 \\
CVEMP IAD & 0.12 & 0.477 & 0.25 & 0.121 \\
OVEMP right ear N1 & -0.48 & 0.233 & 0.08 & 0.858 \\
OVEMP right ear P1 & 0.05 & 0.91 & 0.27 & 0.511 \\
OVEMP left ear N1 & $0.75 *$ & 0.033 & 0.36 & 0.382 \\
OVEMP left ear P1 & 0.1 & 0.821 & 0.03 & 0.952 \\
CVEMP right ear N1 & -0.08 & 0.62 & -0.07 & 0.671 \\
CVEMP right ear P1 & 0.07 & 0.672 & 0.16 & 0.319 \\
CVEMP left ear N1 & 0.22 & 0.18 & $0.42 * *$ & 0.007 \\
CVEMP left ear P1 & -0.04 & 0.823 & 0.16 & 0.315 \\
\hline & $*$ Correlation is significant at the 0.01 level (2-tailed). &
\end{tabular}

\section{Discussion}

This is a comparative cross-sectional study that aimed to explore the role of VEMP in diagnosis of the different kinds of endolymphatic hydrops. The study comprised 40 patients with different types of endolymphatic hydrops. And also the study included 8 patients as a control group.

The study included ten cases with CMD having fluctuating sensorineural hearing loss with tinnitus. Study done by Schaaf et al., that discusses whether low frequency fluctuating hearing loss without labyrinthine vertigo is a genuine disease, actually agrees with our study. They reported that $26 \%$ of CMD patients suffered from vertigo, but only $9 \%$ had developed the typical signs of full-blown MD with the typical labyrinthine vertigo. They concluded that; although almost every patient with MD suffered from recurrent low-frequency hearing loss, only a few patients with recurrent hearing loss developed MD [9].

Additionally, current study included 17 cases with RV. They are a group of patients with a history of more than a single episode of vertigo. RV was defined a disease characterized by more than a single episode of vertigo of duration characteristic of endolymphatic hydrops but without auditory or clinical neurological symptoms or signs. As a distinctive clinical disorder with unknown cause, $\mathrm{RV}$ should be always considered to the patients complaining recurrent episodic vertigo [10].

The study also included 13 with DMD disease. Those with DMD tend to be older than the other two groups. Also, those with CMD tend to be younger than the other two groups. In addition, there was a significant difference in gender prevalence between the three study groups. Male gender was more prevalent in the DMD disease group than the other two groups. This finding agreed with a research article made to study the importance of socioeconomic and environmental factors in $\mathrm{MD}$, a comparison of disease prevalence by gender was made, MD was found to be higher among females with a rate of 84 per 100,000 compared to a rate of 56 per 100,000 among males. Also, the incidence of rate of MD among females was significantly higher than that of males in all the age groups that were older than 41 years old and in the age 11-20 group. The prevalence increased as age increased with the highest prevalence found among the 81 to 90 -year age group [11].

The results of the current study showed that there was significant difference in family history between the three study groups; as it was related to the DMD group and to the RV but not related to the CMD group. This finding agreed with a study made by Requena et al., that discussed familial clustering and familial heterogeneity in Meniere's disease. It was made to estimate the prevalence of familial cases in patients with MD and to identify clinical differences between sporadic and familial MD. It was found that MD was a complex trait and multiple genes and environmental factors contributed to its development. The aggregation of a disease in families was the first observable clue for an underlying genetic susceptibility [ 12]. Thus, therefore a family history of SNHL, migraine or recurrent vertigo in several members of the family would justify a familial investigation of these patients to confirm if any of them fulfill diagnostic criteria for MD [13]. 
Regarding the history of vertiginous attacks in this study, vertigo was more prevalent in the RV group than other groups. Hearing loss was more prevalent in the CMD group. Also, with regard dizziness, the CMD group has less sensation of imbalance or rotation than the two other groups. Headachewas less prevalent in the CMD group than other groups. Aural fullness was less prevalent in the CMD group than other groups. Also, tinnitus was less prevalent in the CMD group than other groups, as shown in Table (1). Huppert et al., in their study of long term Meniere's disease, talked about the effects and course of the long-term of MD. It was found that the frequency of vertigo attacks diminishes within 5-10 years. In the same study it was also found that hearing loss (of about 50-60dB) and vestibular function decrement (of about $35-50 \%$ ) take place mainly in the first 5-10 years of disease. Also, drop attacks were found to occur early or late during the disease, and remission was spontaneous in most cases [14]. Also, VEMP measurements were more likely to be absent in ears with MD and most likely to be absent in Meniere ears that experience drop attacks, as the saccule is the first site to be affected during the disease [15].

In our study, unilateral affection was more in $\mathrm{RV}$ and CMD groups; whereas bilateral affection was more in the DMD group. However, it was found in another study that MD usually starts confined to one ear but it often extended to involve both ears over time [16]. However, Suh et al. (2018) found the prevalence of a bilateral disease was only $5.6 \%$. It was generally agreed that there were often minor symptoms on the other ear, and also that it was rare that both ears were severely impacted. This agrees with our study. Usually, the second ear would be affected only to a mild tinnitus or fullness [17]. On the other hand, bilateral involvement was observed in about one third of MD cases. Saccular hydrops appeared to precede symptoms in bilateral MD patients $[\mathbf{1 8 , 1 9 ]}$.

Our study showed that the DMD group had significantly longer duration than other groups as shown in Table (5). The course of hearing loss seemed to show a considerable tendency to progress over the duration of the illness. However, one review with an overall large number of patients ( $\mathrm{n}=7852$ ) allowed for an assessment of the most probable long-term prognosis of the MD activity. Involvement of the contralateral ear became more frequent the longer the disease lasted [14].

In our cases, after Conventional audiological evaluation, it was showed in the audiogram that there was mild to moderate affection in the DMD group more than the other groups especially at 250, 500, and $1000 \mathrm{HZ}$ frequencies. All cases of DMD and CMD have had SNHL, with only two cases of RV. In a study made on 40 ears, assessing the stage of MD and correlating it with the resultant hearing loss. The audiograms demonstrated lowfrequency hearing loss in 14 ears, high-frequency hearing loss in 7 ears, peak-type hearing loss in 7 ears, and flat-type hearing loss in 12 ears. Lowor hightone hearing loss was found in $83 \%$ of the stage I ears and in $75 \%$ of the stage II ears. In contrast, peak- or flat-type hearing loss was found in $62 \%$ of the stage III ears and in $100 \%$ of the stage IV ears, indicating a significant relationship between the types of hearing loss and the stage of MD [20].

In our study, the speech discrimination scores were significantly lower in the DMD group than both in CMD group and the RV group. That agrees with another study that demonstrated that the score was significantly lower in the DMD group than the other groups. It still matched pure tone thresholds for all patients. Correlations between puretone and speech audiometry were present as in non-Meniere ears and no indications were found of reduced speech discrimination relative to the expectation based on pure-tone loss [21].

In our study, EcochG was carried out using the Golden Triptodes, as shown in Fig. (1). Elevation of SP/AP ratio above the criterion level of abnormality (0.42) according to the established norms of the Ain Shams University Hospital. In our study, non-invasive tympanic membrane electrocochleography was performed for all cases. The mean SP/AP ratio was $>0.40$ in all study groups except for normal ears. The mean left ear sp/ap ratio was significantly different between DMD and CMD groups, and between the DMD and the RV groups. When attempting to diagnose MD, a criterion of 0.42 was set as abnormal value. This criterion was set according to some studies that reported an elevation of SP/AP ratio above the criterion level of abnormality (0.42) was found in $82.85 \%$ of affected ears, as shown in Table (2) [22,23].

In order to fully establish an assessment of the pathophysiology of MD, vestibular testing was performed thoroughly. Classic Video-Nystagmography (VNG) test battery was performed searching for spontaneous, gaze evoked positional and positioning nystagmus. Oculomotor test battery included regular random saccade testing, together with eye tracking and optokinetic tests. Bithermal Caloric test was performed. All cases have had a 
normal VNG spontaneous nystagmus test, VNG gaze test, VNG saccade test, tracking test, optokinetic test and Dix hallpike maneuver. Positional nystagmus and caloric test were comparable between all study groups. However, a functional reduction of about $35-50 \%$ of caloric response was commonly found in the afflicted ear. Only a few patients had a more severe decrement of vestibular function, and the absence of caloric responses was rare [14].

In the current study, both c-VEMP and o-VEMP were carried out in all cases to assess their otolith function. It was found that asymmetric response of c-VEMP was seen in $69.2 \%$ of DMD cases, $82.4 \%$ of RV cases and only in $30 \%$ of CMD cases. This could be explained by Young et al., ion their study about VEMPs in delayed Endolymphatic Hydrops that reported that the IAD ratio of VEMPs correlates with the stage of MD and can be used as another aid to assess the stage of MD [20].

The results of this current study showed that there was a significant difference in c-VEMP between DMD and normal control groups and between the RV group and the normal control group. Asymmetric c-VEMP was significantly more frequent in the RV \& DMD groups than other groups. There was a significant difference in $\mathrm{p} 1$ $\& \mathrm{n} 1$ latencies in right ear between the study groups. The right ear $\mathrm{p} 1$ latency, the difference was between RV and both DMD and CMD. In addition, the right ear $\mathrm{n} 1$ latency, the difference was between RV and both DMD and CMD. Low amplitude of o-VEMP was seen in $84.6 \%$ of DMD cases, $90 \%$ of CMD cases, $88.2 \%$ of RV cases, and only in $25 \%$ of normal cases. Latencies of $n 1$ and p 1 were measured in right and left ear in all cases. There was a significant difference in right ear $\mathrm{p} 1$ $\&$ left ear p1 latencies among the study groups. There was a significant positive strong correlation between age and Echo-G SP/Ap ratio left ear, oVEMP IAD and o-VEMP left ear $\mathrm{n} 1$. In addition, there was a significant positive weak correlation between duration and EcochG SP/AP ratio left ear and c-VEMP left ear $\mathrm{n} 1$. In another study c-VEMP was obtained from both ears of 58 individuals with MD and same number of age and gender matched healthy individuals. The lowest amplitudes were obtained in the affected ears with MD followed by their unaffected ears. The finding of larger asymmetry ratio of c-VEMP in MD might be attributed to the unilateral nature of the pathology. C-VEMP is an ipsilateral response with almost no contribution from the contralateral side, therefore one side might be affected but not the other side. Thereby; producing larger asymmetry between the sides in MD [24-26].

However, it was found that there was little evidence that any type of VEMP is sufficiently sensitive to be useful for diagnosis of MD. It was said that their main role was to diagnose other illnesses that might be confused with MD, and possibly to predict whether gentamicin injection for MD was effective or not [22]. However, a study combining c-VEMP and o-VEMP, it was found that in o-VEMP, there was prolonged latency of $\mathrm{n} 10$ and $\mathrm{p} 15$ as a frequent finding in the study groups. Also, there were differences between the study groups and the control group for most of the wave parameters in combined c-VEMP and oVEMP. Also, it was found that it was lower in patients with MD response. Also, thresholds were higher than in subjects not having MD. The affected ear was more altered than the clinically unaffected ear. Therefore, the air conducted VEMP could be a relevant addition to the diagnosis workup of patients with possible MD. Scarpa et al., in their study about clinical applications of cVEMPs and oVEMPs in patients affected by Meniere's disease; lower response rate, smaller amplitude, and higher threshold of the o-VEMP indicated the pathological disease evidence [27].

\section{Conclusion:}

Vestibular evoked myogenic potential test is a good diagnostic and prognostic tool in identifying different forms of Endolymphatic hydrops. Elevated SP/AP ratio is a good index in identifying Endolymphatic Hydrops in the diseased ear, and can detect early pathology in the contralateral ear and in cochlear Meniere's disease before the full-blown picture of the definitive Meniere's disease prevails.

\section{References}

1- CIUMAN R.R.: Inner ear symptoms and disease: Pathophysiological understanding and therapeutic options. Med. Sci. Monit., 19: 1195-1210, 2013.

2- BERLINGER N.T.: Ménière's disease: New concepts, new treatments. Minn Med., 94: 33-36, 2011.

3- GÜRKOV R., PYYKÖ I., ZOU J. and KENTALA E.: What is Ménière's disease? A contemporary reevaluation of endolymphatic hydrops. J. Neurol., 263 (Suppl 1): S7181, 2016.

4- ROSENGREN S.M., COLEBATCH J.G., YOUNG A.S., GOVENDER S. and WELGAMPOLA M.S.: Vestibular evoked myogenic potentials in practice: Methods, pitfalls and clinical applications. Clin. Neurophysiol. Pract, 4: 47-68, 2019.

5- CURTHOYS I.S., VULOVIC V. and MANZARI L.: Ocular vestibular-evoked myogenic potential (oVEMP) to test utricular function: Neural and oculomotor evidence. Acta. Otorhinolaryngol. Ital, 32: 41-45, 2012. 
6- ROSENGREN S.M., McANGUS TODD N.P. and COLEBATCH J.G.: Vestibular-evoked extraocular potentials produced by stimulation with bone-conducted sound. Clin. Neurophysiol., 116: 1938-1948, 2005.

7- SHEPARD N. and TELIAN S.: Practical Management of the Balance Disorder Patient. San Diego, California: Singular Publishing Group, Inc., 1996.

8- FURMAN J.M. and JACOB R.G.: Jongkees' formula reevaluated: Order effects in the response to alternate binaural bithermal caloric stimulation using closed-loop irrigation. Acta. Otolaryngol., 113: 3-10, 1993.

9- SCHAAF $H$. and HESSE G.: Low frequency fluctuating hearing loss without labyrinthine vertigo-a genuine disease? A follow-up study after 4 and 10 years. HNO, 55 ( 8): 630, 2007.

10- PARK S.N., PARK K.H., IM D.J., KIM J.H., KIM J.Y. and YEO S.W.: RV: Clinical characteristics and efficacy of combination therapy. Journal of the Korean BalanceSociety, 5 (2): 262-268, 2006.

11- SIMO H., YANG S., QU W., PREIS M., NAZZAL M. and BAUGH R.: Ménière's disease: Importance of socioeconomic and environmental factors. American Journal of Otolaryngology, 36 (3): 393-398, 2015.

12- REQUENA T., ESPINOSA-SANCHEZ J.M., CABRERA S., TRINIDAD G., SOTO-VARELA A., SANTOS-PEREZ S. and ARAN I.: Familial clustering and genetic heterogeneity in Meniere's disease. Clinical genetics, 85 (3): 245-252, 2014.

13- LOPEZ-ESCAMEZ J.A., CAREY J., CHUNG W.H., GOEBEL J.A., MAGNUSSON M., MANDALÀ M. and BISDORFF A.: Diagnostic criteria for Ménière's disease. Journal of Vestibular Research, 25 (1): 1-7, 2015.

14- Huppert D., Strupp M., \& Brandt T.: Long-term course of Meniere's disease revisited. Acta. Otolaryngologica, 130 (6): 644-651, 2010.

15- TIMMER F.C., ZHOU G., GUINAN J.J., KUJAWA S.G., HERRMANN B.S. and RAUCH S. D.: Vestibular evoked myogenic potential (VEMP) in patients with Ménière's disease with drop attacks. The Laryngoscope, 116 (5): 776-779, 2006

16- STAHLE J., FRIBERG U. and SVEDBERG A.: Long term progression of Meniere's disease. Acta. Otolaryngol. ( Stockh), 1991: Suppl 485: 75-83, 1991.

17- SUH M.J., JEONG J., KIM H.J., JUNG J. and KIM S.H.: Clinical Characteristics of Bilateral Meniere's Disease in a Single Asian Ethnic Group. Laryngoscope, Oct 3, 2018.doi: 10.1002/lary.27423. [Epub ahead of print].

18- CONTE G., LO RUSSO F.M., CALLONI S.F., SINA C., BAROZZI S., Di BERARDINO F., SCOLA E., PALUM-
BO G., ZANETTI D. and TRIULZI F.M.: MR imaging of endolymphatic hydrops in Ménière's disease: Not all that glitters is gold. Acta otorhinolaryngologica Italica: Organo ufficiale della Societa italiana di otorinolaringologia e chirurgia cervico-facciale, 38 (4): 369-376, 2018. https://doi.org/10.14639/0392-100X-1986.

19- LIN M.Y., TIMMER F.C., ORIEL B.S., ZHOU G., GUINAN J.J., KUJAWA S.G., HERRMANN B.S., MERCHANT S.N. and RAUCH S.D.: Vestibular evoked myogenic potentials (VEMP) can detect asymptomatic saccular hydrops. The Laryngoscope, 116 (6): 987-992, 2006. https://doi.org/10.1097/01.mlg.0000216815.75512.03.

20- YOUNG Y.H., HUANG T.W. and CHENG P.W.: Vestibular evoked myogenic potentials in delayed endolymphatic hydrops. The Laryngoscope, 112 (9): 1623-1626, 2002.

21- KURIOKA T., SANO H., FURUKI S. and YAMASHITA T.: Effects of the Conductive Component of Hearing Loss on Speech Discrimination Ability. The journal of international advanced otology, 16 (1): 93-97, 2020. https://doi.org/10.5152/iao.2020.7870.

22- TIMOTHY C. HAIN: ECochG (ECOG) TESTING (Electrocochleography): available at: https:// www. dizzinessand- balance.com/testing/evoked/ecog.html, 2020.

23- GIBSON W.P.: The Clinical Uses of Electrocochleography. Frontiers in neuroscience, 11: 274, 2017. https://doi.org/ 10.338 9/fnins.2017.00274.

24- ANGELI S.I. and GONCALVES S.: Cervical VEMP tuning changes by Meniere's disease stages. Laryngoscope Investigative Otolaryngology, 4 (5): 543-549, 2019. https://doi.org/10.1002/lio2.309.

25- ZUNIGA M.G., JANKY K.L., SCHUBERT M.C. and CAREY J.P.: Can vestibular-evoked myogenic potentials help differentiate Ménière disease from vestibular migraine? Otolaryngology - head and neck surgery: Official journal of American Academy of Otolaryngology-Head and Neck Surgery, 146 (5): 788-796, 2012. https://doi.org/ 10. $1177 / 0194599811434073$

26- KANTNER C. and GURKOV ROBERT: Characteristics and clinical applications of ocular vestibular evoked myogenic potentials. Hearing Research, 294, 2012. 10. 1016/j.heares.2012.10.008.

27- SCARPA A., GIOACCHINI F.M., CASSANDRO E., TULLI M., RALLI M., RE M. and CASSANDRO C.: Clinical application of cVEMPs and oVEMPs in patients affected by Ménière's disease, vestibular neuritis and benign paroxysmal positional vertigo: A systematic review. Acta otorhinolaryngologica Italica : Organo ufficiale della Societa italiana di otorinolaringologia e chirurgia cervicofacciale, 39 (5): 298-307, 2019. https://doi.org/10.14639/ 0392-100X-2104. 


\section{دورجهاز قياس الجهد العضلى المثار تجهاز الاتزان

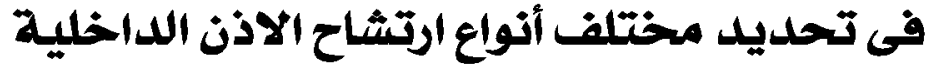

الهدف من هذه الدراسة: هو استكشاف دود الجهود الدهليزية المستحثة للعضل (VEMP) فى تشخيص الأنواع المختلفة من مرض منيير

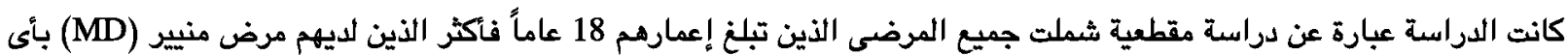

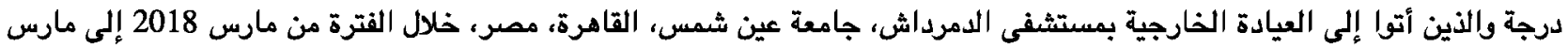

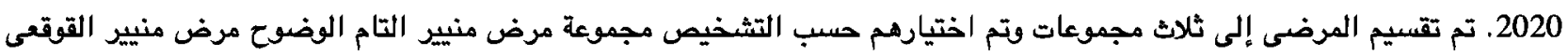

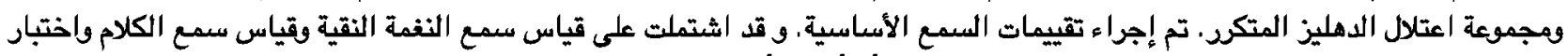

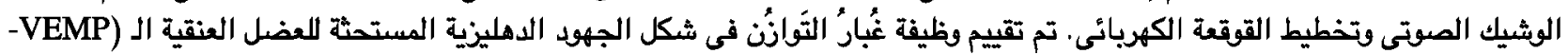

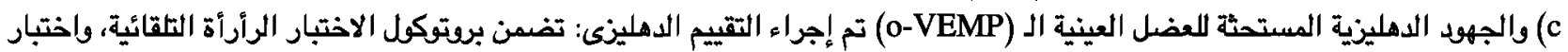

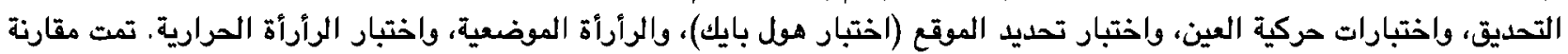
المجموعات الثُلاث لجميع المتفيرات الديموغرافية وكذلك المتفيرات المرضية ونتائج الاختبارات.

كانت العينة الكلية 40 حالة. 13 لديهم مرض منيير التام الوضوح، 10 لديهم مرض منييير القوقعى و 17 لديهم اعتلال الدهليز المتكرد . كان متوسط العمر متفير بين المجموعات (القيمة الاحتمالية أقل من القان

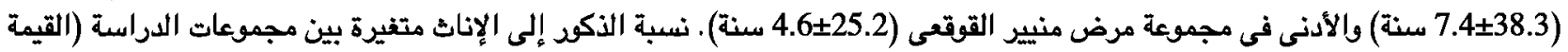

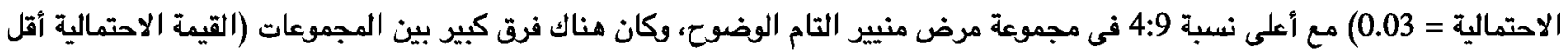

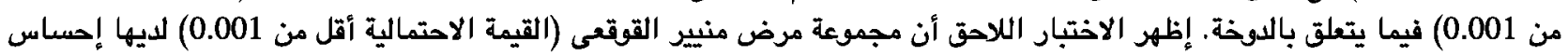
أقل بعدم التوازن أو الدوان من المجموعتين الأخريين.

وأيضاً لقد شوهدت استجابة غير المتماثلة لـ

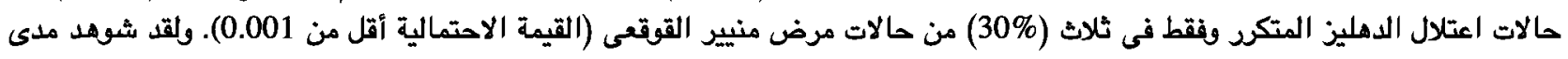

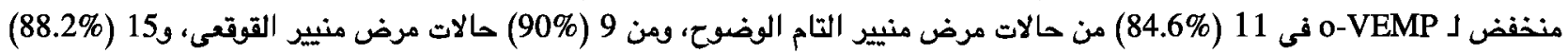

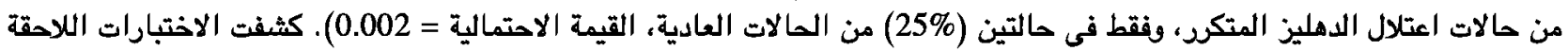

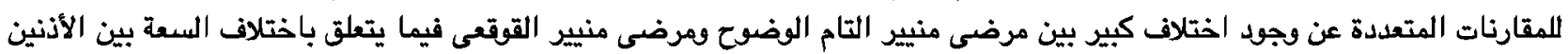

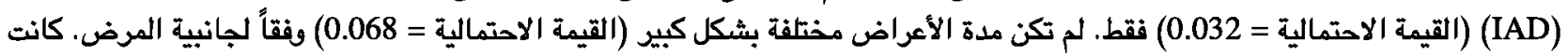

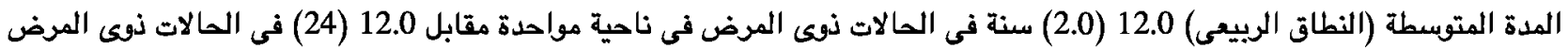

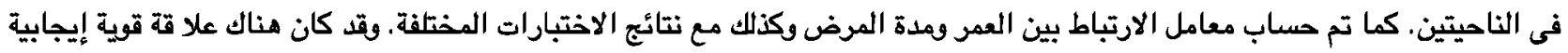

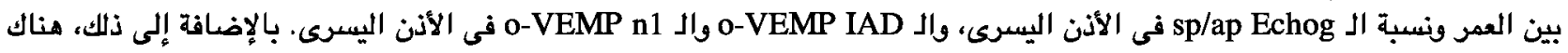
ارتباط ضعيف إيجابى معنوى بين مدة المرض ونسبة sp/ap Echog في الأنن اليسرى ووكذلك الـ c-VEMP للآذن اليسرى.

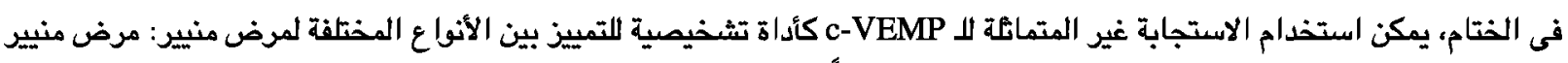

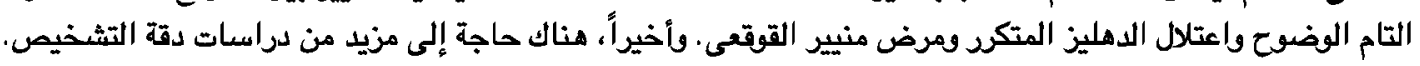

